\title{
Light-by-light scattering in the presence of magnetic fields
}

\author{
R. Baier \\ Faculty of Physics, University of Bielefeld, D-33501 Bielefeld, Germany
}

\author{
A. Rebhan ${ }^{\dagger}$ and M. Wödlinger \\ Institute for Theoretical Physics, Technische Universität Wien, \\ Wiedner Hauptstraße 8-10, A-1040 Vienna, Austria
}

(Received 22 May 2018; published 5 September 2018)

\begin{abstract}
The low-energy light-by-light cross section as determined by the nonlinear Euler-Heisenberg QED Lagrangian is evaluated in the presence of constant magnetic fields in the center-of-mass system of the colliding photons. This cross section has a complicated dependence on directions and polarizations. The overall magnitude decreases as the magnetic field is increased from zero, but this trend is reversed for ultrastrong magnetic fields $B \gtrsim B_{c}$, where the cross section eventually grows quadratically with the magnetic field strength perpendicular to the collision axis. This effect is due to interactions involving the lowest Landau level of virtual Dirac particles; it is absent in scalar QED. An even more dramatic effect is found for virtual charged vector mesons where the one-loop cross section diverges at the critical field strength due to an instability of the lowest Landau level and the possibility of the formation of a superconducting vacuum state. We also discuss (the absence of) implications for the recent observation of light-by-light scattering in heavy-ion collisions.
\end{abstract}

DOI: 10.1103/PhysRevD.98.056001

\section{INTRODUCTION}

Scattering of light by light is a prediction of quantum electrodynamics (QED) that has been first calculated in 1935 , in fact prior to the full development of QED, in the low-energy limit by Euler and Kockel [1,2], and in the ultrarelativistic limit shortly thereafter by Akhiezer, Landau, and Pomeranchuk [3,4]. The former calculations were extended by Heisenberg and Euler [5] who obtained an effective low-energy Lagrangian which includes background electromagnetic fields to all orders in field strength (for historical reviews and references see [6-9]; a short list of further relevant references with regard to applications in light-by-light scattering is given by [10-17]).

In high-energy ultraperipheral collisions of heavy ions (HIC) evidence of the quantum mechanical process of light-by-light scattering has been presented for the first time by the ATLAS Collaboration at the LHC [18], and more recently also by the CMS Collaboration [19]. Light-bylight scattering can be studied through the large (almost)

\footnotetext{
*baier@physik.uni-bielefeld.de

tanton.rebhan@tuwien.ac.at

¥matthias.woedlinger@gmail.com
}

Published by the American Physical Society under the terms of the Creative Commons Attribution 4.0 International license. Further distribution of this work must maintain attribution to the author(s) and the published article's title, journal citation, and DOI. Funded by SCOAP. real photon fluxes available in ultraperipheral hadronhadron, best in lead-lead collisions at LHC.

In the noncentral HICs very strong magnetic fields are created perpendicular to the heavy-ion reaction plane, which, however, decay rapidly, but are still strong at collision time $\tau \simeq 1 \mathrm{fm}$. The field strength has been estimated to reach [20-23]

$$
\begin{aligned}
B / B_{c}(\tau=0 \mathrm{fm}) & \simeq O\left(10^{5}\right) \quad \text { and } \\
B / B_{c}(\tau=0.6 \mathrm{fm}) & \simeq O\left(10^{2}-10^{3}\right),
\end{aligned}
$$

at RHIC for impact parameters $b \simeq 10 \mathrm{fm}$, with the critical magnetic field $B_{c}=\frac{m_{e}^{2}}{e} \approx 0.86 \mathrm{MeV}^{2} \approx 4.4 \times 10^{13} \mathrm{G}$ in terms of the electron mass $m_{e}$. At the LHC the estimated initial value is about a factor of 10 higher (but decays faster).

Motivated by this, the present paper considers $\gamma+\gamma \rightarrow$ $\gamma+\gamma$ scattering in the presence of weak and strong (constant) magnetic fields in the center-of-mass system of the colliding photons, from $B / B_{c} \ll 1$ to $B / B_{c} \gg 1$ (but, parametrically, $B / B_{c} \ll \alpha^{-1 / 2}$ so that higher-loop corrections as well as the effects from dispersion and refraction of light in the magnetic field [24] remain negligible). In the following this process will be studied in detail in the low-energy approximation provided by the Euler-Heisenberg Lagrangian. In this regime, the cross section rises proportional to $\omega^{6} / \mathrm{m}^{8}$ with increasing photon energy $\omega$. At $\omega \sim m$ the cross section reaches its maximum 
value $\propto \alpha^{4} / m^{2}$ and afterwards decays rapidly like $1 / \omega^{2}$ $[3,11,25]$ until the next heavier charged particle starts to contribute according to the Euler-Heisenberg Lagrangian but with a maximum value that is suppressed by the corresponding lower inverse mass squared. After electrons and muons, also scalar charged particles such as pions and kaons contribute, which are described by a variant of the Euler-Heisenberg Lagrangian first obtained by Weisskopf [26]. Also working out the effects of magnetic background fields on virtual scalars, we find that magnetic fields lead to a monotonic decrease of the light-by-light scattering cross section in scalar QED, whereas the lowest Landau level of the Dirac spinors contributes a counteracting effect that dominates at large magnetic fields where it leads to a growing cross section. A theoretically particularly interesting case is given by the Euler-Heisenberg Lagrangian for charged vector bosons [27] for which we find a light-bylight scattering cross section growing with magnetic field strength and diverging at the critical magnetic field where it has been conjectured that a charged vector boson condensate may form [28-31].

As discussed further in the concluding section, relatively more significant effects from magnetic fields are to be expected for lighter particles as they have smaller critical $B_{c}=m^{2} / e$. At least sufficiently below the mass threshold, where the cross section steeply rises with energy, the EulerHeisenberg Lagrangian permits reliable calculations of the effects of magnetic fields on light-by-light scattering.

\section{EFFECTIVE LAGRANGIAN}

The one-loop effective QED Lagrangian for a Dirac particle with charge $e$ and mass $m$ in the presence of electromagnetic background fields with negligible gradients as obtained first by Heisenberg and Euler reads $[5,32,33]$

$$
\begin{aligned}
\mathcal{L}_{\text {spinor }}^{(1)}= & -\frac{1}{8 \pi^{2}} \int_{0}^{\infty} \frac{d s}{s^{3}} \mathrm{e}^{-m^{2} s} \\
& \times\left[(e s)^{2}|\mathcal{G}| \operatorname{coth}\left(e s\left(\sqrt{\mathcal{F}^{2}+\mathcal{G}^{2}}+\mathcal{F}\right)^{\frac{1}{2}}\right)\right. \\
& \left.\times \cot \left(e s\left(\sqrt{\mathcal{F}^{2}+\mathcal{G}^{2}}-\mathcal{F}\right)^{\frac{1}{2}}\right)-\frac{2}{3}(e s)^{2} \mathcal{F}-1\right],
\end{aligned}
$$

where $\mathcal{F}$ and $\mathcal{G}$ denote the Lorentz scalar and pseudoscalar

$$
\begin{gathered}
\mathcal{F}:=\frac{1}{4} F_{\mu \nu} F^{\mu \nu}=\frac{1}{2}\left(\mathbf{B}^{2}-\mathbf{E}^{2}\right), \\
\mathcal{G}:=\frac{1}{4} F_{\mu \nu}{ }^{\star} F^{\mu \nu}=\mathbf{E} \cdot \mathbf{B},
\end{gathered}
$$

that can be built from the field-strength tensor and its dual,

$$
\begin{gathered}
F^{\mu \nu}=\partial^{\mu} A^{\nu}-\partial^{\nu} A^{\mu} \\
{ }^{\star} F^{\mu \nu}=\frac{1}{2} \epsilon^{\mu \nu \alpha \beta} F_{\alpha \beta} .
\end{gathered}
$$

The Maxwell Lagrangian is given by $\mathcal{L}^{(0)}=-\mathcal{F}$.

An equivalent version of (2) is

$$
\begin{aligned}
\mathcal{L}_{\text {spinor }}^{(1)}= & -\frac{1}{8 \pi^{2}} \int_{0}^{\infty} \frac{d s}{s^{3}} \mathrm{e}^{-m^{2} s}\left[(e s)^{2} a b \operatorname{coth}(e s a) \cot (e s b)\right. \\
& \left.-\frac{1}{3}(e s)^{2}\left(a^{2}-b^{2}\right)-1\right]
\end{aligned}
$$

where new variables are introduced ${ }^{1}$

$$
\begin{array}{cl}
a:=\left(\sqrt{\mathcal{F}^{2}+\mathcal{G}^{2}}+\mathcal{F}\right)^{\frac{1}{2}}, & b:=\left(\sqrt{\mathcal{F}^{2}+\mathcal{G}^{2}}-\mathcal{F}\right)^{\frac{1}{2}}, \\
\Rightarrow|\mathcal{G}|=a b, \quad \mathcal{F}=\frac{1}{2}\left(a^{2}-b^{2}\right) .
\end{array}
$$

In terms of the variables $a$ and $b$, the low-energy oneloop effective Lagrangian of QED with Dirac spinors replaced by charged scalars reads [26]

$$
\begin{aligned}
\mathcal{L}_{\text {scalar }}^{(1)}= & \frac{1}{16 \pi^{2}} \int_{0}^{\infty} \frac{d s}{s^{3}} \mathrm{e}^{-m^{2} s}\left[\frac{(e s)^{2} a b}{\sinh (e s a) \sin (e s b)}\right. \\
& \left.+\frac{1}{6}(e s)^{2}\left(a^{2}-b^{2}\right)-1\right],
\end{aligned}
$$

where $m$ is now the mass of the charged scalar particle. This is of potential interest for elastic light-by-light scattering when the photon energy approaches the mass scale of pions.

The Euler-Heisenberg Lagrangian for massive charged vector fields has been obtained in Ref. [27] for the case of a gyromagnetic factor $g=2$, which is carried by the electroweak $W^{ \pm}$gauge bosons and (approximately) also by the $\rho$ meson $[35,36]$. It reads

$$
\begin{aligned}
\mathcal{L}_{\text {vector }}^{(1)}= & 3 \mathcal{L}_{\text {scalar }}^{(1)}+\frac{e^{2}}{4 \pi^{2}} \int_{0}^{\infty} \frac{d s}{s}\left[\mathrm{e}^{-i m^{2} s} a\left(b \frac{\sin (e s a)}{\sinh (e s b)}-a\right)\right. \\
& \left.-\mathrm{e}^{-m^{2} s} b\left(a \frac{\sin (e s b)}{\sinh (e s a)}-b\right)\right]
\end{aligned}
$$

where $m$ on the right-hand side, including the term $3 \mathcal{L}_{\text {scalar }}^{(1)}$, is the mass of the charged vector particle.

For hadronic scalar and vector mesons, the effective Lagrangians (10) and (11) apply as long as they can be

\footnotetext{
${ }^{1}$ Here we follow the conventions used in Refs. [33,34] which differ from the original work of Heisenberg and Euler [5] as well as the review [6] in the notational reversal $a \leftrightarrow b$.
} 
TABLE I. Coefficients $c_{1,2} / C$ and $\hat{c}_{1,2} / C$ with $C=\alpha^{2} / m^{4}$.

\begin{tabular}{lcccc}
\hline \hline & $c_{1} / C$ & $c_{2} / C$ & $\hat{c}_{1} / C$ & $\hat{c}_{2} / C$ \\
\hline Spinor QED & $8 / 45$ & $14 / 45$ & $64 / 315$ & $104 / 315$ \\
Scalar QED & $7 / 90$ & $1 / 90$ & $31 / 315$ & $11 / 315$ \\
Supersymmetric QED & $1 / 3$ & $1 / 3$ & $2 / 5$ & $2 / 5$ \\
Charged massive vector & $29 / 10$ & $27 / 10$ & $-137 / 105$ & $-157 / 105$ \\
\hline \hline
\end{tabular}

treated as pointlike particles, which should be the case at sufficiently large photon wavelength and sufficiently large Larmor radius $r_{q} \propto m_{q} /(e B)$ of the quark constituents, compared to the mesons' charge radii.

In the limit of weak fields, the various Euler-Heisenberg Lagrangians have the form

$$
\mathcal{L}^{(1)}=c_{1} \mathcal{F}^{2}+c_{2} \mathcal{G}^{2}+\ldots,
$$

with $c_{1,2}$ given in Table I. These lowest-order terms are sufficient to obtain the cross section for low-energy lightby-light scattering with zero background fields [1] (see Ref. [15] for detailed results including polarization effects); in the following the corresponding calculations will be generalized to a constant magnetic background field of arbitrary strength.

\section{GEOMETRY AND KINEMATICS}

The scattering amplitude $\mathcal{M}$ for $\gamma\left(k_{1}\right)+\gamma\left(k_{2}\right) \rightarrow$ $\gamma\left(k_{3}\right)+\gamma\left(k_{4}\right)$ is evaluated in the center-of-mass system,

$$
\begin{aligned}
& k_{1}=(\omega, \omega \hat{k}), \quad k_{2}=(\omega,-\omega \hat{k}), \\
& k_{3}=\left(\omega, \omega \hat{k}^{\prime}\right), \quad k_{4}=\left(\omega,-\omega \hat{k}^{\prime}\right) .
\end{aligned}
$$

The scattering plane is defined by

$$
\hat{k}=(1,0,0), \quad \hat{k}^{\prime}=(\cos \theta, \sin \theta, 0) .
$$

For linear polarizations the unit vectors $\hat{\epsilon}_{i}$ and $\hat{\epsilon}_{o}$ denote the directions in and out of the plane of scattering, such that they form a right-handed orthogonal basis with the photon momenta $\hat{k}, \hat{k}^{\prime}$, respectively (see Fig. 1),

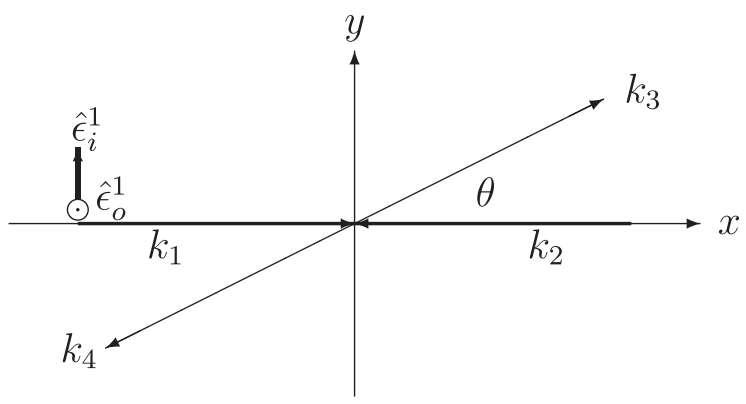

FIG. 1. Kinematics of photon-photon collisions in the centerof-mass system.

$$
\begin{aligned}
& \hat{\epsilon}_{i}^{1}=(0,1,0), \quad \hat{\epsilon}_{o}^{1}=(0,0,1), \\
& \hat{\epsilon}_{i}^{2}=(0,1,0), \quad \hat{\epsilon}_{o}^{2}=(0,0,-1), \\
& \hat{\epsilon}_{i}^{3}=(-\sin \theta, \cos \theta, 0), \quad \hat{\epsilon}_{o}^{3}=(0,0,1), \\
& \hat{\epsilon}_{i}^{4}=(-\sin \theta, \cos \theta, 0), \quad \hat{\epsilon}_{o}^{4}=(0,0,-1) .
\end{aligned}
$$

The radiation field-strength vectors [24] are given by

$$
\begin{aligned}
& \mathbf{f}_{i, o}^{1 \pm}=\omega\left(\hat{k} \wedge \hat{\epsilon}_{i, o}^{1} \pm i \hat{\epsilon}_{i, o}^{1}\right), \\
& \mathbf{f}_{i, o}^{2 \pm}=\omega\left(-\hat{k} \wedge \hat{\epsilon}_{i, o}^{2} \pm i \hat{\epsilon}_{i, o}^{2}\right), \\
& \mathbf{f}_{i, o}^{3 \pm}=\omega\left(\hat{k}^{\prime} \wedge \hat{\epsilon}_{i, o}^{3} \pm i \hat{\epsilon}_{i, o}^{3}\right), \\
& \mathbf{f}_{i, o}^{4 \pm}=\omega\left(-\hat{k}^{\prime} \wedge \hat{\epsilon}_{i, o}^{4} \pm i \hat{\epsilon}_{i, o}^{4}\right) .
\end{aligned}
$$

The external fields are denoted by

$$
\mathbf{F}^{ \pm}=\mathbf{B} \pm i \mathbf{E}
$$

with components $F_{r}^{ \pm}, r=1,2,3$, as for the components $f_{r}^{ \pm}$ of $\mathbf{f}^{ \pm}$.

\section{LIGHT-BY-LIGHT SCATTERING AMPLITUDES AND CROSS SECTIONS}

Following Adler's seminal work on photon splitting in a magnetic field [24] (as reviewed in Sec. 3.4 of Ref. [33]), the matrix element for the scattering $\gamma\left(k_{1}\right)+\gamma\left(k_{2}\right) \rightarrow$ $\gamma\left(k_{3}\right)+\gamma\left(k_{4}\right)$ in the presence of external electromagnetic fields is given by derivatives of the Euler-Heisenberg Lagrangian (2) [or its analogue (10) in scalar QED and (11) for charged vector mesons], which are finally evaluated for finite $\mathbf{B}$ and vanishing $\mathbf{E}=0$. The rather lengthy expression reads

$\mathcal{M}=\left(f_{r}^{1+} \cdot \frac{\partial}{\partial F_{r}^{+}}+f_{r}^{1-} \cdot \frac{\partial}{\partial F_{r}^{-}}\right)\left(f_{s}^{2+} \cdot \frac{\partial}{\partial F_{s}^{+}}+f_{s}^{2-} \cdot \frac{\partial}{\partial F_{s}^{-}}\right)\left(f_{t}^{3+} \cdot \frac{\partial}{\partial F_{t}^{+}}+f_{t}^{3-} \cdot \frac{\partial}{\partial F_{t}^{-}}\right)\left(f_{u}^{4+} \cdot \frac{\partial}{\partial F_{u}^{+}}+f_{u}^{4-} \cdot \frac{\partial}{\partial F_{u}^{-}}\right) \times \mathcal{L}^{(1)}$,

and explicitly, 


$$
\begin{aligned}
\mathcal{M}= & f_{r}^{1+} f_{s}^{2+} f_{t}^{3+} f_{u}^{4+} \frac{\partial^{4} \mathcal{L}^{(1)}}{\partial F_{r}^{+} \partial F_{s}^{+} \partial F_{t}^{+} \partial F_{u}^{+}}+\left(f_{r}^{1+} f_{s}^{2+} f_{t}^{3+} f_{u}^{4-}+f_{r}^{1+} f_{s}^{2+} f_{t}^{4+} f_{u}^{3-}\right. \\
& \left.+f_{r}^{1+} f_{s}^{3+} f_{t}^{4+} f_{u}^{2-}+f_{r}^{2+} f_{s}^{3+} f_{t}^{4+} f_{u}^{1-}\right) \frac{\partial^{4} \mathcal{L}^{(1)}}{\partial F_{r}^{+} \partial F_{s}^{+} \partial F_{t}^{+} \partial F_{u}^{-}}+\left(f_{r}^{1+} f_{s}^{2+} f_{t}^{3-} f_{u}^{4-}+f_{r}^{1+} f_{s}^{3+} f_{t}^{2-} f_{u}^{4-}+f_{r}^{1+} f_{s}^{4+} f_{t}^{2-} f_{u}^{3-}\right. \\
& \left.+f_{r}^{2+} f_{s}^{3+} f_{t}^{1-} f_{u}^{4-}+f_{r}^{3+} f_{s}^{4+} f_{t}^{1-} f_{u}^{2-}+f_{r}^{2+} f_{s}^{4+} f_{t}^{1-} f_{u}^{3-}\right) \frac{\partial^{4} \mathcal{L}^{(1)}}{\partial F_{r}^{+} \partial F_{s}^{+} \partial F_{t}^{-} \partial F_{u}^{-}}+\left(f_{r}^{1-} f_{s}^{2-} f_{t}^{3-} f_{u}^{4+}+f_{r}^{1-} f_{s}^{2-} f_{t}^{4-} f_{u}^{3+}\right. \\
& \left.+f_{r}^{1-} f_{s}^{3-} f_{t}^{4-} f_{u}^{2+}+f_{r}^{2-} f_{s}^{3-} f_{t}^{4-} f_{u}^{1+}\right) \frac{\partial^{4} \mathcal{L}^{(1)}}{\partial F_{r}^{-} \partial F_{s}^{-} \partial F_{t}^{-} \partial F_{u}^{+}}+f_{r}^{1-} f_{s}^{2-} f_{t}^{3-} f_{u}^{4-} \frac{\partial^{4} \mathcal{L}^{(1)}}{\partial F_{r}^{-} \partial F_{s}^{-} \partial F_{t}^{-} \partial F_{u}^{-}} .
\end{aligned}
$$

Next the derivatives with respect to $F_{r}^{ \pm}$are expressed in terms of derivatives $\frac{\partial}{\partial \mathcal{F}}$ and $\frac{\partial}{\partial \mathcal{G}}$, e.g.,

$$
\frac{\partial}{\partial F_{r}^{ \pm}}=\frac{1}{2} F_{r}^{ \pm}\left(\frac{\partial}{\partial \mathcal{F}} \mp i \frac{\partial}{\partial \mathcal{G}}\right)
$$

using

$$
\frac{\partial \mathcal{F}}{\partial F_{r}^{ \pm}}=\frac{1}{2} F_{r}^{ \pm}, \quad \frac{\partial \mathcal{G}}{\partial F_{r}^{ \pm}}= \pm \frac{1}{2 i} F_{r}^{ \pm}
$$

and

$$
\frac{\partial^{2}}{\partial F_{r}^{+} \partial F_{s}^{+}}=\frac{1}{2} \delta_{r s}\left(\frac{\partial}{\partial \mathcal{F}}-i \frac{\partial}{\partial \mathcal{G}}\right)+\frac{1}{4} F_{r}^{+} F_{s}^{+}\left(\frac{\partial^{2}}{\partial \mathcal{F}^{2}}-2 i \frac{\partial^{2}}{\partial \mathcal{F} \partial \mathcal{G}}-\frac{\partial^{2}}{\partial \mathcal{G}^{2}}\right), \quad \text { etc. }
$$

An important typical derivative is

$$
\begin{aligned}
\frac{\partial^{4} \mathcal{L}^{(1)}}{\partial F_{r}^{+} \partial F_{s}^{+} \partial F_{t}^{+} \partial F_{u}^{+}}= & \frac{1}{4}\left(\delta_{r s} \delta_{t u}+\delta_{r t} \delta_{s u}+\delta_{s t} \delta_{r u}\right)\left(\frac{\partial^{2} \mathcal{L}^{(1)}}{\partial \mathcal{F}^{2}}-\frac{\partial^{2} \mathcal{L}^{(1)}}{\partial \mathcal{G}^{2}}\right) \\
& +\frac{1}{8}\left(\delta_{r s} F_{t}^{+} F_{s}^{+}+\delta_{r t} F_{s}^{+} F_{u}^{+}+\delta_{s t} F_{r}^{+} F_{u}^{+}+\delta_{r u} F_{s}^{+} F_{t}^{+}+\delta_{s u} F_{r}^{+} F_{t}^{+}+\delta_{t u} F_{r}^{+} F_{s}^{+}\right) \\
& \times\left(\frac{\partial^{3} \mathcal{L}^{(1)}}{\partial \mathcal{F}^{3}}-3 \frac{\partial^{3} \mathcal{L}^{(1)}}{\partial \mathcal{F} \partial \mathcal{G}^{2}}\right) \\
& +\frac{1}{16} F_{r}^{+} F_{s}^{+} F_{t}^{+} F_{u}^{+}\left(\frac{\partial^{4} \mathcal{L}^{(1)}}{\partial \mathcal{F}^{4}}-6 \frac{\partial^{4} \mathcal{L}^{(1)}}{\partial \mathcal{F}^{2} \partial \mathcal{G}^{2}}+\frac{\partial^{4} \mathcal{L}^{(1)}}{\partial \mathcal{G}^{4}}\right)
\end{aligned}
$$

noting that odd derivatives with respect to $\mathcal{G}$ vanish for $\mathbf{E}=0$, i.e., at $F_{r}^{ \pm}=B_{r}$.

\section{A. Weak magnetic field}

In order to obtain the $O\left(\xi^{2}\right), \xi=B / B_{c}$, correction to the leading-order matrix element $\mathcal{M}_{H E}$ of Eq. (A1) the derivatives of Eq. (B7) enter, i.e.,

$$
\delta \mathcal{M}=\frac{1}{8} \mathcal{M}_{a}\left(\frac{\partial^{3} \mathcal{L}^{(1)}}{\partial \mathcal{F}^{3}}-3 \frac{\partial^{3} \mathcal{L}^{(1)}}{\partial \mathcal{F} \partial \mathcal{G}^{2}}\right)+\frac{1}{8} \mathcal{M}_{b}\left(\frac{\partial^{3} \mathcal{L}^{(1)}}{\partial \mathcal{F}^{3}}+\frac{\partial^{3} \mathcal{L}^{(1)}}{\partial \mathcal{F} \partial \mathcal{G}^{2}}\right)
$$

evaluated at $\mathcal{F}=\mathcal{G}=0$, where

$$
\begin{aligned}
\mathcal{M}_{a}= & \left(\mathbf{f}^{1+} \cdot \mathbf{f}^{4+}\right)\left(\mathbf{f}^{2+} \cdot \mathbf{B}\right)\left(\mathbf{f}^{3+} \cdot \mathbf{B}\right)+\left(\mathbf{f}^{2+} \cdot \mathbf{f}^{4+}\right)\left(\mathbf{f}^{1+} \cdot \mathbf{B}\right)\left(\mathbf{f}^{3+} \cdot \mathbf{B}\right)+\left(\mathbf{f}^{3+} \cdot \mathbf{f}^{4+}\right)\left(\mathbf{f}^{1+} \cdot \mathbf{B}\right)\left(\mathbf{f}^{2+} \cdot \mathbf{B}\right) \\
& +\left(\mathbf{f}^{1+} \cdot \mathbf{f}^{2+}\right)\left(\mathbf{f}^{3+} \cdot \mathbf{B}\right)\left(\mathbf{f}^{4+} \cdot \mathbf{B}\right)+\left(\mathbf{f}^{1+} \cdot \mathbf{f}^{3+}\right)\left(\mathbf{f}^{2+} \cdot \mathbf{B}\right)\left(\mathbf{f}^{4+} \cdot \mathbf{B}\right)+\left(\mathbf{f}^{2+} \cdot \mathbf{f}^{3+}\right)\left(\mathbf{f}^{1+} \cdot \mathbf{B}\right)\left(\mathbf{f}^{4+} \cdot \mathbf{B}\right)+(+\Leftrightarrow-),
\end{aligned}
$$


and

$$
\begin{aligned}
\mathcal{M}_{b}= & \left(\mathbf{f}^{1+} \cdot \mathbf{f}^{2+}\right)\left(\mathbf{f}^{3+} \cdot \mathbf{B}\right)\left(\mathbf{f}^{4-} \cdot \mathbf{B}\right)+\left(\mathbf{f}^{1+} \cdot \mathbf{f}^{3+}\right)\left(\mathbf{f}^{4+} \cdot \mathbf{B}\right)\left(\mathbf{f}^{2-} \cdot \mathbf{B}\right)+\left(\mathbf{f}^{1+} \cdot \mathbf{f}^{3+}\right)\left(\mathbf{f}^{2+} \cdot \mathbf{B}\right)\left(\mathbf{f}^{4-} \cdot \mathbf{B}\right) \\
& +\left(\mathbf{f}^{1+} \cdot \mathbf{f}^{2+}\right)\left(\mathbf{f}^{4+} \cdot \mathbf{B}\right)\left(\mathbf{f}^{3-} \cdot \mathbf{B}\right)+\left(\mathbf{f}^{2+} \cdot \mathbf{f}^{3+}\right)\left(\mathbf{f}^{4+} \cdot \mathbf{B}\right)\left(\mathbf{f}^{1-} \cdot \mathbf{B}\right)+\left(\mathbf{f}^{1+} \cdot \mathbf{f}^{4+}\right)\left(\mathbf{f}^{2+} \cdot \mathbf{B}\right)\left(\mathbf{f}^{3-} \cdot \mathbf{B}\right) \\
& +\left(\mathbf{f}^{1+} \cdot \mathbf{f}^{4+}\right)\left(\mathbf{f}^{3+} \cdot \mathbf{B}\right)\left(\mathbf{f}^{2-} \cdot \mathbf{B}\right)+\left(\mathbf{f}^{2+} \cdot \mathbf{f}^{3+}\right)\left(\mathbf{f}^{1+} \cdot \mathbf{B}\right)\left(\mathbf{f}^{4-} \cdot \mathbf{B}\right)+\left(\mathbf{f}^{3+} \cdot \mathbf{f}^{4+}\right)\left(\mathbf{f}^{2+} \cdot \mathbf{B}\right)\left(\mathbf{f}^{1-} \cdot \mathbf{B}\right) \\
& +\left(\mathbf{f}^{2+} \cdot \mathbf{f}^{4+}\right)\left(\mathbf{f}^{3+} \cdot \mathbf{B}\right)\left(\mathbf{f}^{1-} \cdot \mathbf{B}\right)+\left(\mathbf{f}^{2+} \cdot \mathbf{f}^{4+}\right)\left(\mathbf{f}^{1+} \cdot \mathbf{B}\right)\left(\mathbf{f}^{3-} \cdot \mathbf{B}\right)+\left(\mathbf{f}^{3+} \cdot \mathbf{f}^{4+}\right)\left(\mathbf{f}^{1+} \cdot \mathbf{B}\right)\left(\mathbf{f}^{2-} \cdot \mathbf{B}\right) \\
& +\left(\mathbf{f}^{3-} \cdot \mathbf{f}^{4-}\right)\left(\mathbf{f}^{1+} \cdot \mathbf{B}\right)\left(\mathbf{f}^{2+} \cdot \mathbf{B}\right)+\left(\mathbf{f}^{2-} \cdot \mathbf{f}^{4-}\right)\left(\mathbf{f}^{1+} \cdot \mathbf{B}\right)\left(\mathbf{f}^{3+} \cdot \mathbf{B}\right)+\left(\mathbf{f}^{2-} \cdot \mathbf{f}^{3-}\right)\left(\mathbf{f}^{1+} \cdot \mathbf{B}\right)\left(\mathbf{f}^{4+} \cdot \mathbf{B}\right) \\
& +\left(\mathbf{f}^{1-} \cdot \mathbf{f}^{4-}\right)\left(\mathbf{f}^{2+} \cdot \mathbf{B}\right)\left(\mathbf{f}^{3+} \cdot \mathbf{B}\right)+\left(\mathbf{f}^{1-} \cdot \mathbf{f}^{2-}\right)\left(\mathbf{f}^{3+} \cdot \mathbf{B}\right)\left(\mathbf{f}^{4+} \cdot \mathbf{B}\right)+\left(\mathbf{f}^{1-} \cdot \mathbf{f}^{3-}\right)\left(\mathbf{f}^{2+} \cdot \mathbf{B}\right)\left(\mathbf{f}^{4+} \cdot \mathbf{B}\right)+(+\Leftrightarrow-) .
\end{aligned}
$$

With

$$
\mathcal{L}^{(1)}=c_{1} \mathcal{F}^{2}+c_{2} \mathcal{G}^{2}-\hat{c}_{1} \frac{\mathcal{F}^{3}}{B_{c}^{2}}-\hat{c}_{2} \frac{\mathcal{F} \mathcal{G}^{2}}{B_{c}^{2}} \pm \ldots
$$

and the explicit values derived in Appendix B 1 and tabulated in Table I, the amplitudes for the linear polarizations in and out of the collision plane read ${ }^{2}$

$$
\begin{aligned}
\frac{\mathcal{M}_{\text {oоoo }}}{\omega^{4}}= & 4 c_{1}\left(3+\cos ^{2} \theta\right)+\left\{\begin{array}{r}
-30 \hat{c}_{1} \\
-30 \hat{c}_{1} \\
-18 \hat{c}_{1}+16 \hat{c}_{2}
\end{array}\right\} \xi^{2} \\
& +\left\{\begin{array}{r}
6 \hat{c}_{1} \\
-42 \hat{c}_{1} \\
-6 \hat{c}_{1}
\end{array}\right\} \xi^{2} \cos ^{2} \theta,
\end{aligned}
$$$$
\frac{\mathcal{M}_{i i i i}}{\omega^{4}}=4 c_{1}\left(3+\cos ^{2} \theta\right)+\left\{\begin{array}{r}
-18 \hat{c}_{1}+4 \hat{c}_{2} \\
-18 \hat{c}_{1}+4 \hat{c}_{2} \\
-66 \hat{c}_{1}
\end{array}\right\} \xi^{2}
$$$$
+\left\{\begin{array}{r}
-6 \hat{c}_{1}-4 \hat{c}_{2} \\
-6 \hat{c}_{1}+12 \hat{c}_{2} \\
-6 \hat{c}_{1}
\end{array}\right\} \xi^{2} \cos ^{2} \theta
$$

$$
\begin{aligned}
\frac{\mathcal{M}_{\text {ooii }}}{\omega^{4}}= & -8 c_{1}+4 c_{2}\left(1+\cos ^{2} \theta\right)+\left\{\begin{array}{r}
12 \hat{c}_{1}-6 \hat{c}_{2} \\
24 \hat{c}_{1}-2 \hat{c}_{2} \\
24 \hat{c}_{1}-14 \hat{c}_{2}
\end{array}\right\} \xi^{2} \\
& +\left\{\begin{array}{c}
2 \hat{c}_{2} \\
-14 \hat{c}_{2} \\
-2 \hat{c}_{2}
\end{array}\right\} \xi^{2} \cos ^{2} \theta
\end{aligned}
$$

\footnotetext{
${ }^{2}$ For vanishing magnetic background fields, this agrees with the results given in Ref. [15] except that a factor $-i$ has been absorbed in the definition of $\mathcal{M}$ as done also in Ref. [24].
}

$$
\begin{aligned}
\frac{\mathcal{M}_{\text {iioo }}}{\omega^{4}}= & -8 c_{1}+4 c_{2}\left(1+\cos ^{2} \theta\right)+\left\{\begin{array}{r}
24 \hat{c}_{1}-2 \hat{c}_{2} \\
12 \hat{c}_{1}-6 \hat{c}_{2} \\
24 \hat{c}_{1}-14 \hat{c}_{2}
\end{array}\right\} \xi^{2} \\
& +\left\{\begin{array}{r}
-12 \hat{c}_{1}-2 \hat{c}_{2} \\
12 \hat{c}_{1}-10 \hat{c}_{2} \\
-2 \hat{c}_{2}
\end{array}\right\} \xi^{2} \cos ^{2} \theta
\end{aligned}
$$

$$
\frac{\mathcal{M}_{\text {oioi,ioio }}}{\omega^{4}}=4\left(c_{1}+c_{2}\right)(1+\cos \theta)
$$$$
+2\left(c_{2}-c_{1}\right)\left(3+\cos ^{2} \theta\right)+\left\{\begin{array}{c}
3 \hat{c}_{1}-9 \hat{c}_{2} \\
3 \hat{c}_{1}-9 \hat{c}_{2} \\
9 \hat{c}_{1}-19 \hat{c}_{2}
\end{array}\right\} \xi^{2}
$$$$
+\left\{\begin{array}{r}
-6 \hat{c}_{1}-2 \hat{c}_{2} \\
-12 \hat{c}_{1}-4 \hat{c}_{2} \\
-12 \hat{c}_{1}-4 \hat{c}_{2}
\end{array}\right\} \xi^{2} \cos \theta
$$$$
+\left\{\begin{array}{r}
3\left(\hat{c}_{1}+\hat{c}_{2}\right) \\
9 \hat{c}_{1}-11 \hat{c}_{2} \\
3 \hat{c}_{1}-\hat{c}_{2}
\end{array}\right\} \xi^{2} \cos ^{2} \theta
$$

$$
\mathcal{M}_{\text {oiio,iooi }}=\left.\mathcal{M}_{\text {oioi,ioio }}\right|_{\cos \theta \rightarrow-\cos \theta},
$$

where the three entries within the curly brackets refer to $\mathbf{B}$ pointing in $x, y$, and $z$ direction, respectively. For such $\mathbf{B}$, the remaining amplitudes with an odd number of $i$ or $o$ polarizations vanish identically.

While we refrain from listing the unwieldy general case of oblique orientations of the magnetic field for all amplitudes, Appendix B 2 gives the general weak-field result for the resulting unpolarized cross section. The resulting total unpolarized cross section reads 


$$
\begin{aligned}
& \sigma(\gamma \gamma \rightarrow \gamma \gamma)^{\text {unpol }} \\
&= \frac{1}{2} \int d \Omega \frac{d \sigma^{\text {unpol }}}{d \Omega} \\
&= \frac{7\left(3 c_{1}^{2}-2 c_{1} c_{2}+3 c_{2}^{2}\right) \omega^{6}}{20 \pi} \\
&+\frac{\omega^{6}}{15 \pi} \frac{B_{\|}^{2}}{B_{c}^{2}}\left(-57 c_{1} \hat{c}_{1}+18 \hat{c}_{1} c_{2}+10 c_{1} \hat{c}_{2}-23 c_{2} \hat{c}_{2}\right) \\
&+\frac{\omega^{6}}{120 \pi} \frac{B_{\perp}^{2}}{B_{c}^{2}}\left(-717 c_{1} \hat{c}_{1}+243 \hat{c}_{1} c_{2}+233 c_{1} \hat{c}_{2}-391 c_{2} \hat{c}_{2}\right),
\end{aligned}
$$

where $B_{\|}$is the magnetic field component parallel to the collision axis of the photons and $B_{\perp}$ the part orthogonal to it. For spinor QED this yields

$$
\begin{aligned}
& \sigma(\gamma \gamma \rightarrow \gamma \gamma)_{\text {spinor }}^{\text {unpol }} \\
& \quad=\frac{973 \alpha^{4} \omega^{6}}{10125 \pi m^{8}}\left[1-\frac{38224 B_{\|}^{2}+65602 B_{\perp}^{2}}{20433 B_{c}^{2}}+O\left(\xi^{4}\right)\right],
\end{aligned}
$$

and for QED with a charged scalar field instead of a Dirac spinor one has

$$
\begin{aligned}
& \sigma(\gamma \gamma \rightarrow \gamma \gamma)_{\text {scalar }}^{\text {unpol }} \\
& \quad=\frac{119 \alpha^{4} \omega^{6}}{20250 \pi m^{8}}\left[1-\frac{11294 B_{\|}^{2}+16802 B_{\perp}^{2}}{2499 B_{c}^{2}}+O\left(\xi^{4}\right)\right] .
\end{aligned}
$$

Scalar QED is relevant for light-by-light scattering at energies below the peak in the cross section produced by muons, since there charged pions also start to contribute. It is moreover particularly interesting in that it highlights the effects of the magnetic moments in spinor QED: In scalar QED, the total cross section is only about $6 \%$ of the result in spinor QED. (Even with two charged scalars so that scalar QED has the same number of degrees of freedom (d.o.f.), the cross section is less than a quarter of that of spinor QED.) This is reflected by the relatively small coefficients $c_{2}$ and $\hat{c}_{2}$ associated with the terms involving the square of the pseudoscalar $\mathcal{G}=\frac{1}{4} F_{\mu \nu}{ }^{\star} F^{\mu \nu}$ (see Table I). Moreover, turning on a (subcritical) magnetic field decreases the total cross section more than twice as strongly as is the case in spinor QED. In fact, as will be shown below, the limit of strong magnetic fields is dominated by the lowest Landau level of Dirac spinors which eventually leads to an increase of the cross section.

As an aside we note that supersymmetric QED, which in Ref. [15] has been shown to have particularly simple polarization patterns, gives the slightly simpler result $\sigma(\gamma \gamma \rightarrow \gamma \gamma)_{\mathrm{sQED}}^{\mathrm{unpol}}=\frac{7 \alpha^{4} \omega^{6}}{45 \pi m^{8}}\left[1-\frac{104 B_{\|}^{2}+158 B_{\perp}^{2}}{35 B_{c}^{2}}+O\left(\xi^{4}\right)\right]$.

Of potential interest to light-by-light scattering are also charged vector bosons, in particular at photon energies between the pion and the $\rho$ meson mass scales. In hadronic contributions to light-by-light scattering, which is a critical ingredient in calculations of the anomalous magnetic moment of muons [37], it is usually assumed that at the scale of the $\rho$ meson one can switch to quark d.o.f. [11]. However, light-by-light scattering through virtual quarks differs quite strongly from the one through virtual vector bosons. In Table I we have also given the coefficients in the expansion of the Euler-Heisenberg Lagrangian resulting from vector mesons with gyromagnetic factor $g=2$ $[27,38,39]$ corresponding to non-Abelian vector bosons as well as to vector mesons [36] (see also [35]). The interactions due to the magnetic moment of the vector mesons turn out to have the effect of enhancing the lightby-light cross section already in the weak-field limit:

$$
\begin{aligned}
& \sigma(\gamma \gamma \rightarrow \gamma \gamma)_{\text {vector }}^{\text {unpol }} \\
& \quad=\frac{2751 \alpha^{4} \omega^{6}}{250 \pi m^{8}}\left[1+\frac{211846 B_{\|}^{2}+318298 B_{\perp}^{2}}{173313 B_{c}^{2}}+O\left(\xi^{4}\right)\right],
\end{aligned}
$$

which is a stark difference to both scalar and spinor QED. As we shall discuss presently, this difference becomes even more pronounced as $\xi$ approaches unity, where one enters a regime with possible vector boson condensation [29-31]. Furthermore, already at vanishing magnetic field, the total cross section for a charged vector boson is very much larger than that produced by three scalar d.o.f. of the same mass, to wit, by a factor of $3537 / 17 \approx 208.06$, underlining the importance of the magnetic moment of the virtual particles in light-by-light scattering.

\section{B. Intermediate field strength}

For $\xi=B / B_{c} \gtrsim 0.5$, the weak-field expansion breaks down and one has to resort to numerical evaluations of the integral representations of the various derivatives of $\mathcal{L}_{c}$ appearing in (18).

Our numerical results are shown in Figs. 2 and 3 for magnetic fields perpendicular and parallel to the collision axis, respectively, where the former case is the one of potential relevance to HIC. In these plots we compare the result for spinor QED and scalar QED, where in the latter case two charged scalar particles are assumed so that the difference between the two results is entirely due to the additional interactions of the magnetic moment carried by Dirac spinors. Also given are the weak-field limits up to order $\xi^{2}$ derived above, which are seen to become inaccurate around $\xi \simeq 0.5$. 
$B_{\perp}$

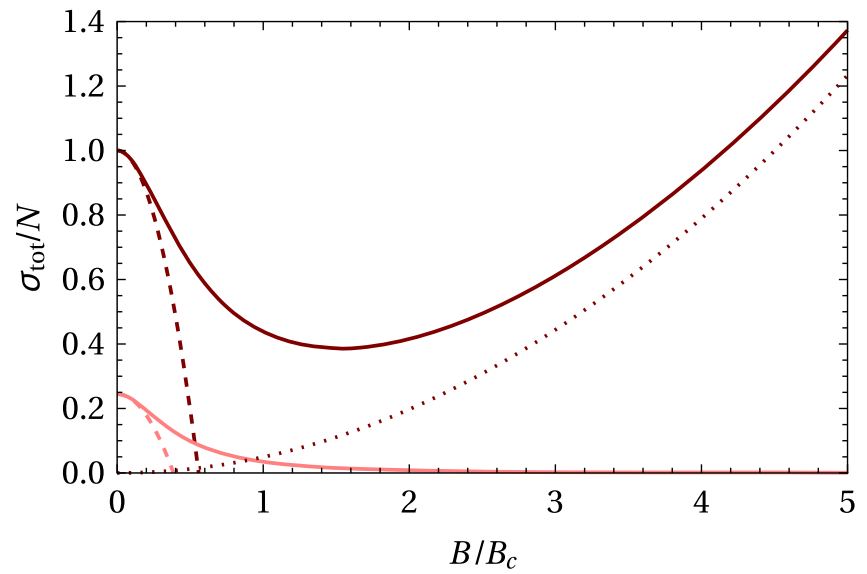

FIG. 2. Total cross section for unpolarized photons as a function of $\xi=B / B_{c}$ with magnetic field perpendicular to the collision axis for spinor QED (dark-red line) and for QED with two charged scalars (light-red line), both normalized to the total cross section of spinor QED at zero magnetic field. The weakfield result to order $\xi^{2}$ is given by the corresponding dashed lines. The strong-field result (45) for spinor QED is given by the dotted black line.

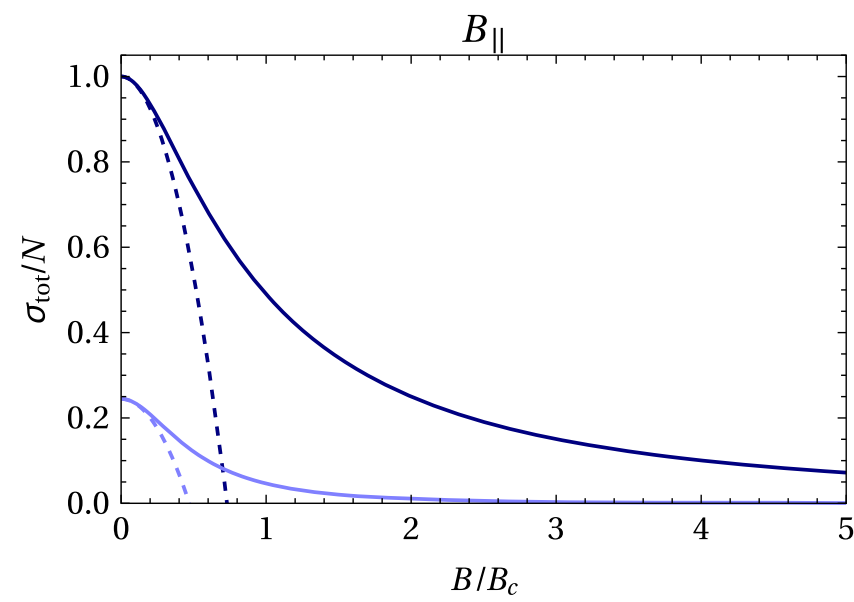

FIG. 3. Same as Fig. 2 but with magnetic field parallel to the collision axis (now dark-blue and light-blue coloring for spinor and scalar QED, respectively).

For larger $\xi$, the results for scalar QED are seen to tend to zero rapidly $\left(\sim \xi^{-4}\right.$ for $\left.\xi \gg 1\right)$, whereas the spinor QED result for the case of perpendicular magnetic field has a minimum at $\xi \simeq 1.5$ after which it grows quadratically with $\xi$.

Further details that show up in differential cross sections are displayed in Appendix C.

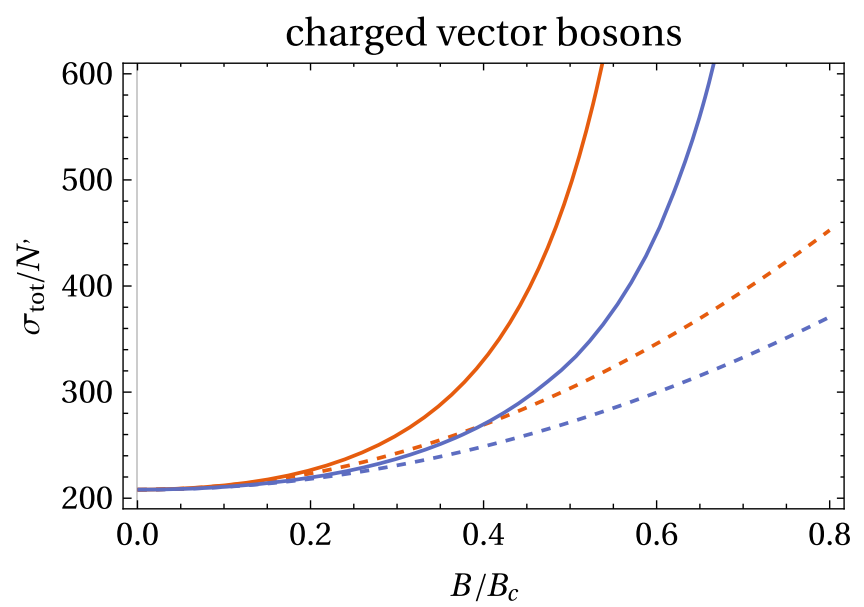

FIG. 4. Total unpolarized light-by-light scattering cross section for virtual charged vector bosons with $g=2$ as a function of $\xi=B / B_{c}$ with the magnetic field perpendicular (red lines) and longitudinal (blue lines) to the collision axis (dashed lines give the corresponding weak-field results). In order to highlight the effects of the magnetic moment of the charged vector bosons, the normalization constant $N^{\prime}$ is chosen as the $B=0$ result for three charged scalars of the same charge and mass, which is a factor $3537 / 17 \approx 208.06$ smaller than for one massive charged vector boson.

In the case of QED with charged vector bosons, for which the total cross section with magnetic field perpendicular or longitudinal to the collision axis is evaluated in Fig. 4, we find an increase which is quadratic in $\xi$ for small $\xi$ and which dramatically accelerates for larger $\xi$ with a divergence at $\xi=1$. In fact, at $\xi>1$ the lowest Landau level of a charged vector with $g=2$ becomes tachyonic, corresponding to the conjectured condensation of the charged vector bosons to form a superconducting vacuum [29-31]. As explained in Appendix B 3, the calculation of the light-by-light scattering cross section through the Euler-Heisenberg Lagrangian is valid only for $\omega^{2} / m^{2} \ll 1-\xi$ so that the singularity is never reached.

\section{Strong magnetic field}

In the limit $\xi=B / B_{c} \gg 1$ (but parametrically $\left.\xi^{2} \ll 1 / \alpha\right)$ the dominant contribution in spinor QED comes from the derivative $\partial^{4} \mathcal{L}^{(1)} / \partial \mathcal{G}^{4}$ at $\mathcal{G}=0$, so that e.g.,

$$
\left.\frac{\partial^{4} \mathcal{L}^{(1)}}{\partial F_{r}^{+} \partial F_{s}^{+} \partial F_{t}^{+} \partial F_{u}^{+}} \rightarrow \frac{1}{16} B_{r} B_{s} B_{t} B_{u} \frac{\partial^{4} \mathcal{L}^{(1)}}{\partial \mathcal{G}^{4}}\right|_{\mathcal{G}=0} .
$$

Thus the matrix element in leading order of a strong magnetic field becomes 


$$
\begin{aligned}
\mathcal{M} /\left(\frac{1}{16} \frac{\partial^{4} \mathcal{L}^{(1)}}{\partial \mathcal{G}^{4}}\right)= & \left(\mathbf{f}^{1+} \cdot \mathbf{B}\right)\left(\mathbf{f}^{2+} \cdot \mathbf{B}\right)\left(\mathbf{f}^{3+} \cdot \mathbf{B}\right)\left(\mathbf{f}^{4+} \cdot \mathbf{B}\right)+\left(\mathbf{f}^{1-} \cdot \mathbf{B}\right)\left(\mathbf{f}^{2-} \cdot \mathbf{B}\right)\left(\mathbf{f}^{3-} \cdot \mathbf{B}\right)\left(\mathbf{f}^{4-} \cdot \mathbf{B}\right) \\
& -\left(\mathbf{f}^{1+} \cdot \mathbf{B}\right)\left(\mathbf{f}^{2+} \cdot \mathbf{B}\right)\left(\mathbf{f}^{3+} \cdot \mathbf{B}\right)\left(\mathbf{f}^{4-} \cdot \mathbf{B}\right)-\left(\mathbf{f}^{1+} \cdot \mathbf{B}\right)\left(\mathbf{f}^{2+} \cdot \mathbf{B}\right)\left(\mathbf{f}^{3-} \cdot \mathbf{B}\right)\left(\mathbf{f}^{4+} \cdot \mathbf{B}\right) \\
& -\left(\mathbf{f}^{1+} \cdot \mathbf{B}\right)\left(\mathbf{f}^{2-} \cdot \mathbf{B}\right)\left(\mathbf{f}^{3+} \cdot \mathbf{B}\right)\left(\mathbf{f}^{4+} \cdot \mathbf{B}\right)-\left(\mathbf{f}^{1-} \cdot \mathbf{B}\right)\left(\mathbf{f}^{2+} \cdot \mathbf{B}\right)\left(\mathbf{f}^{3+} \cdot \mathbf{B}\right)\left(\mathbf{f}^{4+} \cdot \mathbf{B}\right) \\
& +\left(\mathbf{f}^{1+} \cdot \mathbf{B}\right)\left(\mathbf{f}^{2+} \cdot \mathbf{B}\right)\left(\mathbf{f}^{3-} \cdot \mathbf{B}\right)\left(\mathbf{f}^{4-} \cdot \mathbf{B}\right)+\left(\mathbf{f}^{1+} \cdot \mathbf{B}\right)\left(\mathbf{f}^{2-} \cdot \mathbf{B}\right)\left(\mathbf{f}^{3+} \cdot \mathbf{B}\right)\left(\mathbf{f}^{4-} \cdot \mathbf{B}\right) \\
& +\left(\mathbf{f}^{1+} \cdot \mathbf{B}\right)\left(\mathbf{f}^{2-} \cdot \mathbf{B}\right)\left(\mathbf{f}^{3-} \cdot \mathbf{B}\right)\left(\mathbf{f}^{4+} \cdot \mathbf{B}\right)+\left(\mathbf{f}^{1-} \cdot \mathbf{B}\right)\left(\mathbf{f}^{2+} \cdot \mathbf{B}\right)\left(\mathbf{f}^{3+} \cdot \mathbf{B}\right)\left(\mathbf{f}^{4-} \cdot \mathbf{B}\right) \\
& +\left(\mathbf{f}^{1-} \cdot \mathbf{B}\right)\left(\mathbf{f}^{2-} \cdot \mathbf{B}\right)\left(\mathbf{f}^{3+} \cdot \mathbf{B}\right)\left(\mathbf{f}^{4+} \cdot \mathbf{B}\right)+\left(\mathbf{f}^{1-} \cdot \mathbf{B}\right)\left(\mathbf{f}^{2+} \cdot \mathbf{B}\right)\left(\mathbf{f}^{3-} \cdot \mathbf{B}\right)\left(\mathbf{f}^{4+} \cdot \mathbf{B}\right) \\
& -\left(\mathbf{f}^{1-} \cdot \mathbf{B}\right)\left(\mathbf{f}^{2-} \cdot \mathbf{B}\right)\left(\mathbf{f}^{3-} \cdot \mathbf{B}\right)\left(\mathbf{f}^{4+} \cdot \mathbf{B}\right)-\left(\mathbf{f}^{1-} \cdot \mathbf{B}\right)\left(\mathbf{f}^{2-} \cdot \mathbf{B}\right)\left(\mathbf{f}^{3+} \cdot \mathbf{B}\right)\left(\mathbf{f}^{4-} \cdot \mathbf{B}\right) \\
& -\left(\mathbf{f}^{1-} \cdot \mathbf{B}\right)\left(\mathbf{f}^{2+} \cdot \mathbf{B}\right)\left(\mathbf{f}^{3-} \cdot \mathbf{B}\right)\left(\mathbf{f}^{4-} \cdot \mathbf{B}\right)-\left(\mathbf{f}^{1+} \cdot \mathbf{B}\right)\left(\mathbf{f}^{2-} \cdot \mathbf{B}\right)\left(\mathbf{f}^{3-} \cdot \mathbf{B}\right)\left(\mathbf{f}^{4-} \cdot \mathbf{B}\right) .
\end{aligned}
$$

An amplitude with polarization vectors $\hat{\epsilon}^{1,2,3,4}$ [cf. Eq. (15)] is given by

$$
\mathcal{M}=\omega^{4} \frac{\partial^{4} \mathcal{L}^{(1)}}{\partial^{4} \mathcal{G}} \prod_{I=1}^{4} \hat{\epsilon}^{I} \cdot \mathbf{B}=\frac{32 \alpha^{2}}{15}\left(\frac{\omega}{m}\right)^{4} \xi \prod_{I=1}^{4} \hat{\epsilon}^{I} \cdot \hat{\mathbf{B}}+O\left(\xi^{0}\right)
$$

where $\hat{\mathbf{B}}$ is the unit vector in the direction of $\mathbf{B}$. For example, when $\mathbf{B}$ points in the $z$ direction, i.e., orthogonal to the scattering plane, the only nonvanishing amplitude for linear polarizations is

$$
\left.\mathcal{M}_{\text {oooo }}\right|_{B_{x}=B_{y}=0}=\frac{32 \alpha^{2}}{15}\left(\frac{\omega}{m}\right)^{4} \xi+O\left(\xi^{0}\right),
$$

which is $\theta$ independent; when $\mathbf{B}$ points in the $y$ direction, i.e., in the scattering plane and orthogonal to the incoming photons, the only nonvanishing amplitude is

$$
\left.\mathcal{M}_{i i i i}\right|_{B_{x}=B_{z}=0}=\frac{32 \alpha^{2}}{15}\left(\frac{\omega}{m}\right)^{4} \xi \cos ^{2} \theta+O\left(\xi^{0}\right) \text {, }
$$

which vanishes for outgoing photon momenta in the direction of $\mathbf{B}$.

The low-energy unpolarized cross section averaged over initial and summed over final polarizations for $\xi \gg 1$ and arbitrary orientation of $\mathbf{B}$ reads

$\frac{d \sigma_{\text {spinor }}^{\text {unpol }}}{d \Omega}=\frac{1}{(16 \pi)^{2} \omega^{2}} \frac{1}{4}|\mathcal{M}|^{2}=\frac{\alpha^{4} \omega^{6}}{225 \pi^{2} m^{8}} \xi^{2} \sin ^{4} \beta \sin ^{4} \beta^{\prime}$,

where $\beta$ is the angle between $\mathbf{B}$ and the direction of the incoming photon $\hat{k}$, and $\beta^{\prime}$ is the angle between $\mathbf{B}$ and the outgoing direction $\hat{k}^{\prime}$. Notice that this differential cross section has the form of the square of a dipole radiation pattern, with emission maximal in the plane orthogonal to the magnetic field.
The resulting unpolarized total cross section for $\xi \gg 1$ is

$$
\sigma(\gamma \gamma \rightarrow \gamma \gamma)_{\text {spinor }}^{\text {unpol }}=\frac{1}{2} \int d \Omega \frac{d \sigma^{\text {unpol }}}{d \Omega}=\frac{16 \alpha^{4} \omega^{6}}{3375 \pi m^{8}} \xi^{2} \sin ^{4} \beta
$$

Thus, for ultra-strong magnetic fields, the EulerHeisenberg photon scattering cross section grows quadratically with field strength. As shown in Appendix B 3, this feature is absent in scalar QED. It is entirely due to the magnetic moments of the virtual Dirac spinors which in the lowest Landau level lead to a cancellation of magnetic interaction energy.

\section{DISCUSSION}

In this paper we have investigated the effect of sizable background magnetic fields on the light-by-light scattering cross section in QED with charged scalar, spinor, or massive vector fields. We have found that the one-loop contribution of charged scalars to the Euler-Heisenberg Lagrangian lead to a strong suppression of the light-bylight scattering cross section for $B \gtrsim 0.5 B_{c}$. For spinor QED, the cross section initially also decreases with increasing magnetic field, but this trend is reversed at $B \simeq 1.5 B_{c}$ after which the cross section grows quadratically with $B$.

Although at HIC the magnetic field reaches extremely large values with respect to the critical one in terms of the electron mass $m_{e}$, so that the light-by-light scattering cross section would become correspondingly large, this applies only at low photon energies $\omega \lesssim m_{e}$.

In the recent ATLAS measurement [18] of light-by-light scattering the characteristic energy of the scattered photons is in the range of several $\mathrm{GeV}$, with peak values of the background magnetic field $B \sim 10^{5} \mathrm{MeV}^{2}$. Because the cross section decreases as $\alpha^{4} / \omega^{2}$ for $\omega \gg m$, only massive loops can contribute effects due to external magnetic fields. The critical magnetic field corresponding to the bottom and the charm quarks with mass $m_{b} \approx 4.2 \mathrm{GeV}$ and 
$m_{c} \approx 1.25 \mathrm{GeV}$ is $B_{c}\left(m_{b}\right) \sim 6 \times 10^{7} \mathrm{MeV}^{2}$ and $B_{c}\left(m_{c}\right) \sim$ $5 \times 10^{6} \mathrm{MeV}^{2}$, respectively. Effects from external magnetic fields at $\omega \lesssim m_{b}$ are therefore completely negligible. For energies $\omega \lesssim m_{c}$, such effects would still be tiny; noticeable effects on light-by-light scattering would seem to require photon energies $\omega \lesssim 0.1 \mathrm{GeV}$, at or below the maximal contribution to the cross section from virtual muons for which $B_{c}\left(m_{\mu}\right) \sim 4 \times 10^{4} \mathrm{MeV}^{2}$. However, with respect to the corresponding time scale $\omega^{-1}$, the magnetic field in HIC is then probably decaying too fast to leave measurable effects.

A case of particular theoretical interest is that of charged $\rho$ mesons which have an unstable lowest Landau level at $B \geq B_{c}\left(m_{\rho}\right) \sim 2 \times 10^{6} \mathrm{MeV}^{2}$, where a superconducting vacuum state formed by a condensate of $\rho^{ \pm}$mesons has been conjectured to arise [29]. ${ }^{3}$ In this paper we have also determined the contribution of charged vector mesons to light-by-light scattering for photon energies $\omega \lesssim m_{\rho}$ as determined by the corresponding Euler-Heisenberg Lagrangian derived in [27]. This turns out to be enhanced by relatively large numerical prefactors compared to scalar and spinor loops. Moreover, the cross section grows as the magnetic field strength is increased from zero. Unfortunately, even the peak values of the magnetic field reached in HIC would give only effects below the percent level to light-by-light scattering cross sections from virtual $\rho$ mesons (if the latter are included at all despite the large width of the $\rho$ meson).

\section{ACKNOWLEDGMENTS}

The authors would like to thank Maxim Chernodub, Dima Kharzeev, Massimiliano Procura, and Vladimir Skalozub for useful discussions of the case of charged vector mesons.

\section{APPENDIX A: MATRIX ELEMENT FOR B $=0$}

For completeness the matrix element for $\gamma\left(k_{1}\right)+$ $\gamma\left(k_{2}\right) \rightarrow \gamma\left(k_{3}\right)+\gamma\left(k_{4}\right)$ for vanishing external fields is given to fix the notation (see e.g., $[10,11])$,

$$
\begin{aligned}
\mathcal{M}= & \frac{c_{1}}{2} \mathcal{M}_{1}-\frac{c_{2}}{2} \mathcal{M}_{2}, \\
\mathcal{M}_{1}= & \left(\mathbf{f}^{1+} \cdot \mathbf{f}^{2+}\right)\left(\mathbf{f}^{3+} \cdot \mathbf{f}^{4+}\right)+\left(\mathbf{f}^{1+} \cdot \mathbf{f}^{3+}\right)\left(\mathbf{f}^{2+} \cdot \mathbf{f}^{4+}\right)+\left(\mathbf{f}^{2+} \cdot \mathbf{f}^{3+}\right)\left(\mathbf{f}^{1+} \cdot \mathbf{f}^{4+}\right)+\left(\mathbf{f}^{1-} \cdot \mathbf{f}^{2-}\right)\left(\mathbf{f}^{3-} \cdot \mathbf{f}^{4-}\right) \\
& +\left(\mathbf{f}^{1-} \cdot \mathbf{f}^{3-}\right)\left(\mathbf{f}^{2-} \cdot \mathbf{f}^{4-}\right)+\left(\mathbf{f}^{2-} \cdot \mathbf{f}^{3-}\right)\left(\mathbf{f}^{1-} \cdot \mathbf{f}^{4-}\right)+\left(\mathbf{f}^{1+} \cdot \mathbf{f}^{2+}\right)\left(\mathbf{f}^{3-} \cdot \mathbf{f}^{4-}\right)+\left(\mathbf{f}^{1+} \cdot \mathbf{f}^{3+}\right)\left(\mathbf{f}^{2-} \cdot \mathbf{f}^{4-}\right) \\
& +\left(\mathbf{f}^{1+} \cdot \mathbf{f}^{4+}\right)\left(\mathbf{f}^{2-} \cdot \mathbf{f}^{3-}\right)+\left(\mathbf{f}^{2+} \cdot \mathbf{f}^{3+}\right)\left(\mathbf{f}^{1-} \cdot \mathbf{f}^{4-}\right)+\left(\mathbf{f}^{3+} \cdot \mathbf{f}^{4+}\right)\left(\mathbf{f}^{1-} \cdot \mathbf{f}^{2-}\right)+\left(\mathbf{f}^{2+} \cdot \mathbf{f}^{4+}\right)\left(\mathbf{f}^{1-} \cdot \mathbf{f}^{3-}\right),
\end{aligned}
$$

and

$$
\begin{aligned}
\mathcal{M}_{2}= & \left(\mathbf{f}^{1+} \cdot \mathbf{f}^{2+}\right)\left(\mathbf{f}^{3+} \cdot \mathbf{f}^{4+}\right)+\left(\mathbf{f}^{1+} \cdot \mathbf{f}^{3+}\right)\left(\mathbf{f}^{2+} \cdot \mathbf{f}^{4+}\right)+\left(\mathbf{f}^{2+} \cdot \mathbf{f}^{3+}\right)\left(\mathbf{f}^{1+} \cdot \mathbf{f}^{4+}\right)+\left(\mathbf{f}^{1-} \cdot \mathbf{f}^{2-}\right)\left(\mathbf{f}^{3-} \cdot \mathbf{f}^{4-}\right) \\
& +\left(\mathbf{f}^{1-} \cdot \mathbf{f}^{3-}\right)\left(\mathbf{f}^{2-} \cdot \mathbf{f}^{4-}\right)+\left(\mathbf{f}^{2-} \cdot \mathbf{f}^{3-}\right)\left(\mathbf{f}^{1-} \cdot \mathbf{f}^{4-}\right)-\left(\mathbf{f}^{1+} \cdot \mathbf{f}^{2+}\right)\left(\mathbf{f}^{3-} \cdot \mathbf{f}^{4-}\right)-\left(\mathbf{f}^{1+} \cdot \mathbf{f}^{3+}\right)\left(\mathbf{f}^{2-} \cdot \mathbf{f}^{4-}\right)-\left(\mathbf{f}^{1+} \cdot \mathbf{f}^{4+}\right)\left(\mathbf{f}^{2-} \cdot \mathbf{f}^{3-}\right) \\
& -\left(\mathbf{f}^{2+} \cdot \mathbf{f}^{3+}\right)\left(\mathbf{f}^{1-} \cdot \mathbf{f}^{4-}\right)-\left(\mathbf{f}^{3+} \cdot \mathbf{f}^{4+}\right)\left(\mathbf{f}^{1-} \cdot \mathbf{f}^{2-}\right)-\left(\mathbf{f}^{2+} \cdot \mathbf{f}^{4+}\right)\left(\mathbf{f}^{1-} \cdot \mathbf{f}^{3-}\right) .
\end{aligned}
$$

For comparison the cross section is quoted (for references and a detailed evaluation see [15]). For low energies $\omega \leq m$ it is

$\frac{d \sigma^{\mathrm{unpol}}}{d \Omega}=\frac{\omega^{6}}{64 \pi^{2}}\left(3 c_{1}^{2}-2 c_{1} c_{2}+3 c_{2}^{2}\right)\left(3+\cos ^{2} \theta\right)^{2}$.

In the high energy limit it decreases like

$$
\frac{d \sigma^{\mathrm{unpol}}}{d \Omega} \sim \frac{\alpha^{4}}{\omega^{2}}
$$

beyond its maximum at $\omega \simeq 1.5 \mathrm{~m}$ [4].

\footnotetext{
${ }^{3}$ Evidence in favor of this scenario from lattice gauge theory has been presented in $[40,41]$; see however $[42,43]$.
}

\section{APPENDIX B: EXPANSIONS FOR WEAK AND STRONG BACKGROUND FIELDS}

\section{Weak-field limit of $\mathcal{L}^{(1)}$}

The weak-field limit of the Euler-Heisenberg Lagrangian for spinors and scalars, Eqs. (7) and (10), respectively, up to order $\xi^{2}=\left(\frac{B}{B_{c}}\right)^{2}$ is obtained by starting with the Taylor expansion for

$\operatorname{coth} z=\frac{1}{z}+\frac{z}{3}-\frac{z^{3}}{45} \pm \ldots, 1 / \sinh z=\frac{1}{z}-\frac{z}{6}+\frac{7 z^{3}}{360} \mp \ldots$

and

$\cot z=\frac{1}{z}-\frac{z}{3}-\frac{z^{3}}{45}-\ldots, \quad 1 / \sin z=\frac{1}{z}+\frac{z}{6}+\frac{7 z^{3}}{360}+\ldots$ 
leading in terms of the variables $a$ and $b$ to

$$
\begin{aligned}
\mathcal{L}_{\text {spinor }}^{(1)}= & \frac{e^{4}}{8 \pi^{2}} \int_{0}^{\infty} d s s \mathrm{e}^{-m^{2} s}\left[\frac{a^{4}+5 a^{2} b^{2}+b^{4}}{45}\right. \\
& \left.-(e s)^{2} \frac{2 a^{6}+7 a^{4} b^{2}-7 a^{2} b^{4}-2 b^{6}}{945}\right] \pm \ldots,
\end{aligned}
$$

$$
\begin{aligned}
\mathcal{L}_{\text {scalar }}^{(1)}= & \frac{e^{4}}{16 \pi^{2}} \int_{0}^{\infty} d s s \mathrm{e}^{-m^{2} s}\left[\frac{7 a^{4}-10 a^{2} b^{2}+7 b^{4}}{360}\right. \\
& \left.-(e s)^{2} \frac{31 a^{6}-49 a^{4} b^{2}+49 a^{2} b^{4}-31 b^{6}}{15120}\right] \pm \ldots
\end{aligned}
$$

For $a<m^{2}$ one can write the Euler-Heisenberg Lagrangian for charged vector bosons (11) also as

$$
\begin{aligned}
\mathcal{L}_{\text {vector }}^{(1)} & =-\frac{1}{16 \pi^{2}} \int_{0}^{\infty} \frac{d s}{s^{3}} \mathrm{e}^{-m^{2} s}\left[(e s)^{2} a b \frac{1-2 \cosh (2 e s a)-2 \cos (2 e s b)}{\sinh (e s a) \sin (e s b)}+\frac{7}{2}(e s)^{2}\left(a^{2}-b^{2}\right)+3\right] \\
& =\frac{e^{4}}{16 \pi^{2}} \int_{0}^{\infty} d s s \mathrm{e}^{-m^{2} s}\left[\frac{29 a^{4}+50 a^{2} b^{2}+29 b^{4}}{40}+(e s)^{2} \frac{137 a^{6}+217 a^{4} b^{2}-217 a^{2} b^{4}-137 b^{6}}{15120}\right] \pm \ldots
\end{aligned}
$$

After performing the $s$ integration one obtains

$$
\mathcal{L}^{(1)}=c_{1} \mathcal{F}^{2}+c_{2} \mathcal{G}^{2}-\hat{c}_{1} \frac{\mathcal{F}^{3}}{B_{c}^{2}}-\hat{c}_{2} \frac{\mathcal{F} \mathcal{G}^{2}}{B_{c}^{2}}+\ldots
$$

in terms of the variables $\mathcal{F}$ and $\mathcal{G}$ [cf. Eq. (9)], with coefficients as given in Table I. ${ }^{4}$ (Supersymmetric QED has $\mathcal{L}_{\text {sQED }}^{(1)}=\mathcal{L}_{\text {spinor }}^{(1)}+2 \mathcal{L}_{\text {scalar }}^{(1)}$. $)$

The contributions to the light-by-light scattering amplitudes to order $\xi^{2}$ are obtained with $\mathcal{F} \rightarrow \frac{B^{2}}{2}$ and $\mathcal{G} \rightarrow 0$ from

$$
\begin{aligned}
\frac{\partial^{2} \mathcal{L}^{(1)}}{\partial \mathcal{F}^{2}} & =2 c_{1}-3 \hat{c}_{1} \xi^{2}+\ldots, \quad \frac{\partial^{2} \mathcal{L}^{(1)}}{\partial \mathcal{G}^{2}}=2 c_{2}-\hat{c}_{2} \xi^{2}+\ldots \\
\frac{\partial^{3} \mathcal{L}^{(1)}}{\partial \mathcal{F}^{3}} & =-\frac{6 \hat{c}_{1}}{B_{c}^{2}}+\ldots, \quad \frac{\partial^{3} \mathcal{L}^{(1)}}{\partial \mathcal{F} \partial \mathcal{G}^{2}}=-\frac{2 \hat{c}_{2}}{B_{c}^{2}}+\ldots
\end{aligned}
$$

\section{General expression for the unpolarized cross section to order $\xi^{2}$}

In Sec. IVA the weak-field limit of the scattering amplitudes for linearly polarized photons has been given for three cases of the orientation of the magnetic background field. The case of general orientation is rather unwieldy for the various polarized cross sections, but a comparatively compact expression is obtained for the unpolarized cross section, which reads

$$
\begin{aligned}
\frac{d \sigma^{\text {unpol }}}{d \Omega}= & \frac{\omega^{6}}{256 \pi^{2}}\left(3 c_{1}^{2}-2 c_{1} c_{2}+3 c_{2}^{2}\right)(7+\cos 2 \theta)^{2}+\frac{\omega^{6}}{512 \pi^{2} B_{c}^{2}}\left\{B _ { x } ^ { 2 } \left[-1017 c_{1} \hat{c}_{1}+327 \hat{c}_{1} c_{2}+161 c_{1} \hat{c}_{2}-391 c_{2} \hat{c}_{2}\right.\right. \\
& \left.+4 c_{2}\left(15 \hat{c}_{1}+\hat{c}_{2}\right) \cos 2 \theta-4 c_{1}\left(33 \hat{c}_{1}+7 \hat{c}_{2}\right) \cos 2 \theta-3 c_{2}\left(\hat{c}_{1}-\hat{c}_{2}\right) \cos 4 \theta-c_{1}\left(3 \hat{c}_{1}+5 \hat{c}_{2}\right) \cos 4 \theta\right] \\
& +B_{y}^{2}\left[-1563 c_{1} \hat{c}_{1}+501 \hat{c}_{1} c_{2}+459 c_{1} \hat{c}_{2}-813 c_{2} \hat{c}_{2}-4 c_{1}\left(177 \hat{c}_{1}-73 \hat{c}_{2}\right) \cos 2 \theta+12 c_{2}\left(21 \hat{c}_{1}-37 \hat{c}_{2}\right) \cos 2 \theta\right. \\
& \left.+c_{2}\left(15 \hat{c}_{1}-23 \hat{c}_{2}\right) \cos 4 \theta-c_{1}\left(33 \hat{c}_{1}-17 \hat{c}_{2}\right) \cos 4 \theta\right]+B_{z}^{2}\left[-1875 c_{1} \hat{c}_{1}+657 \hat{c}_{1} c_{2}+667 c_{1} \hat{c}_{2}-1073 c_{2} \hat{c}_{2}\right. \\
& \left.-420 c_{1} \hat{c}_{1} \cos 2 \theta+12 c_{2}\left(9 \hat{c}_{1}-17 \hat{c}_{2}\right) \cos 2 \theta+100 c_{1} \hat{c}_{2} \cos 2 \theta-9 c_{1} \hat{c}_{1} \cos 4 \theta+3 c_{2}\left(\hat{c}_{1}-\hat{c}_{2}\right) \cos 4 \theta+c_{1} \hat{c}_{2} \cos 4 \theta\right] \\
& \left.+B_{x} B_{y}\left[\left(516 c_{1} \hat{c}_{1}-156 \hat{c}_{1} c_{2}-276 c_{1} \hat{c}_{2}+396 c_{2} \hat{c}_{2}\right) \sin 2 \theta+\left(30 c_{1} \hat{c}_{1}-18 \hat{c}_{1} c_{2}-22 c_{1} \hat{c}_{2}+26 c_{2} \hat{c}_{2}\right) \sin 4 \theta\right]\right\}+O\left(\xi^{4}\right) .
\end{aligned}
$$

For the particularly important case of spinor QED this yields

\footnotetext{
${ }^{4}$ The results obtained for charged vector mesons are in agreement with those given in [44].
} 


$$
\begin{aligned}
\frac{d \sigma_{\text {spinor }}^{\text {unpol }}}{d \Omega}= & \frac{\alpha^{4} \omega^{6}}{64(45 \pi)^{2} m^{8}}\left\{139(7+\cos 2 \theta)^{2}-\frac{2}{7} \frac{B_{x}^{2}}{B_{c}^{2}}(41441+1956 \cos 2 \theta+251 \cos 4 \theta)\right. \\
& -\frac{2}{7} \frac{B_{y}^{2}}{B_{c}^{2}}(72075+33764 \cos 2 \theta+1425 \cos 4 \theta)-\frac{2}{7} \frac{B_{z}^{2}}{B_{c}^{2}}(86167+20756 \cos 2 \theta+341 \cos 4 \theta) \\
& \left.+\frac{4}{7} \frac{B_{x} B_{y}}{B_{c}^{2}}(14730 \sin 2 \theta+587 \sin 4 \theta)\right\}+O\left(\xi^{4}\right)
\end{aligned}
$$

\section{Strong-field limit of $\partial^{4} \mathcal{L}^{(1)} / \partial \mathcal{G}^{4}$}

In spinor QED, the asymptotic behavior for $\xi=\frac{B}{B_{c}} \gg 1$ and $\mathcal{G} \rightarrow 0$ is determined by the terms in the integrand of the Euler-Heisenberg Lagrangian (2) proportional to

$\operatorname{coth}\left(e s\left(\sqrt{\mathcal{F}^{2}+\mathcal{G}^{2}}+\mathcal{F}\right)^{1 / 2}\right)=\operatorname{coth}(e s a) \equiv \operatorname{coth} t$

with $t=e s a \rightarrow e s B$.

Performing the Taylor expansion

$$
\begin{aligned}
\mathcal{G} \cot (e s b) & =\frac{\mathcal{G}}{e s b}\left[1-\frac{(e s b)^{2}}{3}-\frac{(e s b)^{4}}{45}+\ldots\right] \\
& \simeq \frac{B}{e s}\left[1-\frac{(e s)^{2}}{3 B^{2}} \mathcal{G}^{2}-\frac{(e s)^{4}}{45 B^{4}} \mathcal{G}^{4}+\ldots\right],
\end{aligned}
$$

for $\mathcal{G} \rightarrow 0$ with $b \simeq \mathcal{G} / B$, es $b \simeq e s \mathcal{G} / B$, one obtains

$$
\begin{aligned}
\frac{\partial^{4} \mathcal{L}_{\text {spinor }}^{(1)}}{\partial \mathcal{G}^{4}} & \simeq \frac{1}{8 \pi^{2}} \frac{24}{45 B^{3}} \int_{0}^{\infty} \frac{d s}{s^{3}}(e s)^{5} \mathrm{e}^{-m^{2} s} \operatorname{coth}(e s B) \\
& \simeq \frac{e^{2}}{8 \pi^{2} B^{6}} \frac{8}{15} \int_{0}^{\infty} d t \mathrm{e}^{-t / \xi} t^{2} \operatorname{coth} t
\end{aligned}
$$

i.e., asymptotically for $\xi \gg 1$,

$$
\frac{\partial^{4} \mathcal{L}_{\text {spinor }}^{(1)}}{\partial \mathcal{G}^{4}} \simeq \frac{e^{2}}{8 \pi^{2} B^{6}} \frac{16}{15} \xi^{3}
$$

in agreement with the results derived in [45]. Since in the scattering amplitude (41) this is combined with four powers of the magnetic field, one has $\mathcal{M} \propto B$ in the limit of ultrastrong fields.

This result is, however, a special feature of spinor QED. The Euler-Heisenberg Lagrangian for scalar QED (10) as obtained originally by Weisskopf [26] differs by the absence of the interaction term $\frac{e}{2} \sigma_{\mu \nu} F^{\mu \nu}$. This has the effect that instead of the functions $\operatorname{coth}(e s a)$ and $\cot (e s b)$ in (2) one has $1 / \sinh (e s a)$ and $1 / \sin (e s b)$ [6]. In place of (B12) one obtains

$$
\int_{0}^{\infty} d t \mathrm{e}^{-t / \xi} t^{2} / \sinh t=\frac{7}{2} \zeta(3)+O\left(\xi^{-1}\right) \approx 4.207 \ldots+O\left(\xi^{-1}\right)
$$

in the large- $\xi$ limit. This leads to contributions to $\mathcal{M}$ that are suppressed $\propto B^{-2}$ at large $B$.

As is particularly clear in the derivation of the EulerHeisenberg Lagrangian due to Schwinger [32], the interaction with a spin magnetic moment $g \mu_{B} / 2$ contributes the factor $\cosh ($ gesa/2) $\cos ($ gesb/2), which for $g=2 \mathrm{com}$ pensates the exponential decay of $1 / \sinh ($ esa), corresponding to the fact that then the magnetic interaction energy of a Dirac spinor cancels in the lowest Landau level. This in fact suggests that also for Dirac spinors the rise of the photon-photon scattering amplitude $\sim \xi$ will be modified eventually by higher-order effects at $\xi \gtrsim \alpha^{-1}$, when $(g-2) e B \gtrsim m^{2}$. However, already at the parametrically smaller order $\xi \gtrsim \alpha^{-1 / 2}$ our calculations would need to be modified by including dispersion effects from nontrivial indices of refraction and birefringence [24].

In the case of the Euler-Heisenberg Lagrangian for charged vector bosons with $g=2$ obtained in [27] the effects of the magnetic moment at high magnetic fields are even more dramatic. The magnetic interaction energy, which leads to a modified mass

$m^{2} \rightarrow m_{\mathrm{eff}}^{2}=m^{2}+\left(2 n-g s_{z}+1\right) e B, \quad n \geq 0$,

for spin projection $s_{z}$ along the magnetic field, now reduces the effective mass of the lowest Landau level, such that it becomes imaginary for $e B>m^{2}$, corresponding to the potential instability of the vacuum against formation of a superconducting condensate of charged vector bosons [29].

In the light-by-light scattering cross section as derived from the Euler-Heisenberg Lagrangian, the vanishing of the effective mass in the lowest Landau level leads to a divergence, shown in Fig. 5, indicating a breakdown of perturbation theory. Indeed, the range of validity of the calculation changes from $\omega \ll m$ to $\omega \ll m_{\text {eff }}$, i.e., $\omega^{2} / m^{2} \ll 1-\xi$, for charged vector bosons with $g=2$.

The divergence of the light-by-light scattering amplitude caused by charged vector bosons can be traced to the spin contribution in (11). Expanding the integrand on the righthand side of (11) in powers of $b$, the integral can be evaluated with result 
charged vector bosons

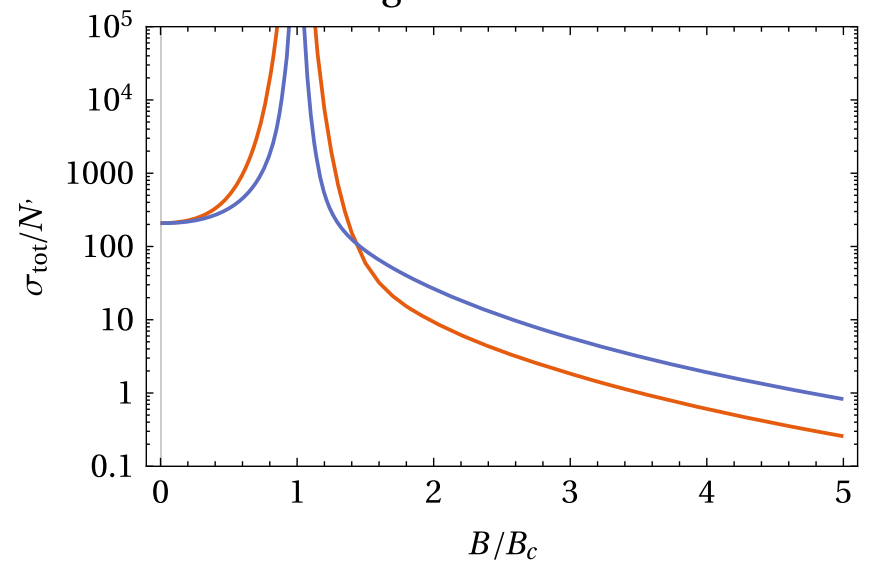

FIG. 5. Total unpolarized light-by-light scattering cross section for virtual charged vector bosons with $g=2$ as a function of $\xi=B / B_{c}$ with the magnetic field perpendicular (red lines) and longitudinal (blue lines) to the collision axis, normalized as in Fig. 4 , but now plotted up to $\xi=5$ in logarithmic scale. At the critical field strength, $B=B_{c}$, the cross section diverges and perturbation theory breaks down. The latter is also the case for $B>B_{c}$, as the Lagrangian density has an imaginary part, signaling instability against vector meson condensation.

$$
\begin{aligned}
& \mathcal{L}_{\text {vector }}^{(1)}-3 \mathcal{L}_{\text {scalar }}^{(1)} \\
& =\frac{1}{8 \pi^{2}}\left[2(e a)^{2}-(e a)^{2} \ln \left(1-\frac{(e a)^{2}}{m^{4}}\right)+e a m^{2} \ln \frac{m^{2}-e a}{m^{2}+e a}\right] \\
& \quad+\frac{(e a)^{2}}{24 \pi\left(m^{4}-(e a)^{2}\right)}(e b)^{2}+\frac{7(e a)^{2}\left(3 m^{4}+(e a)^{2}\right)}{720 \pi^{2}\left(m^{4}-(e a)^{2}\right)^{3}}(e b)^{4} \\
& \quad+O\left(b^{6}\right),
\end{aligned}
$$

where $m^{2}$ is to be understood as having an infinitesimal negative imaginary part, $m^{2} \rightarrow m^{2}-i \epsilon$, when $e a \geq m^{2}$. Evidently, there is a singularity at $e a=m^{2}$ which leads to a multiple pole in the scattering amplitude at $B=B_{c}$. For $e a>m^{2}$, a finite result is obtained, but the Lagrangian then has an imaginary part at $b=0$, i.e., for a purely magnetic background field, which corresponds to the possibility [29-31] of the decay of the vacuum into a superconducting state of condensed charged vector bosons.

\section{APPENDIX C: POLAR DIAGRAMS FOR UNPOLARIZED CROSS SECTIONS}

In Fig. 6 we display the unpolarized differential cross section of spinor QED $^{5}$ as a function of the strength of the background magnetic field for three orientations of the magnetic field with respect to the scattering plane (chosen as the $x y$ plane, see Fig. 1). For small to medium field strength, the cross section decreases with $B / B_{c}$ in all directions, but at high field strength it rises again in directions orthogonal to $\mathbf{B}$.
$B_{x}$
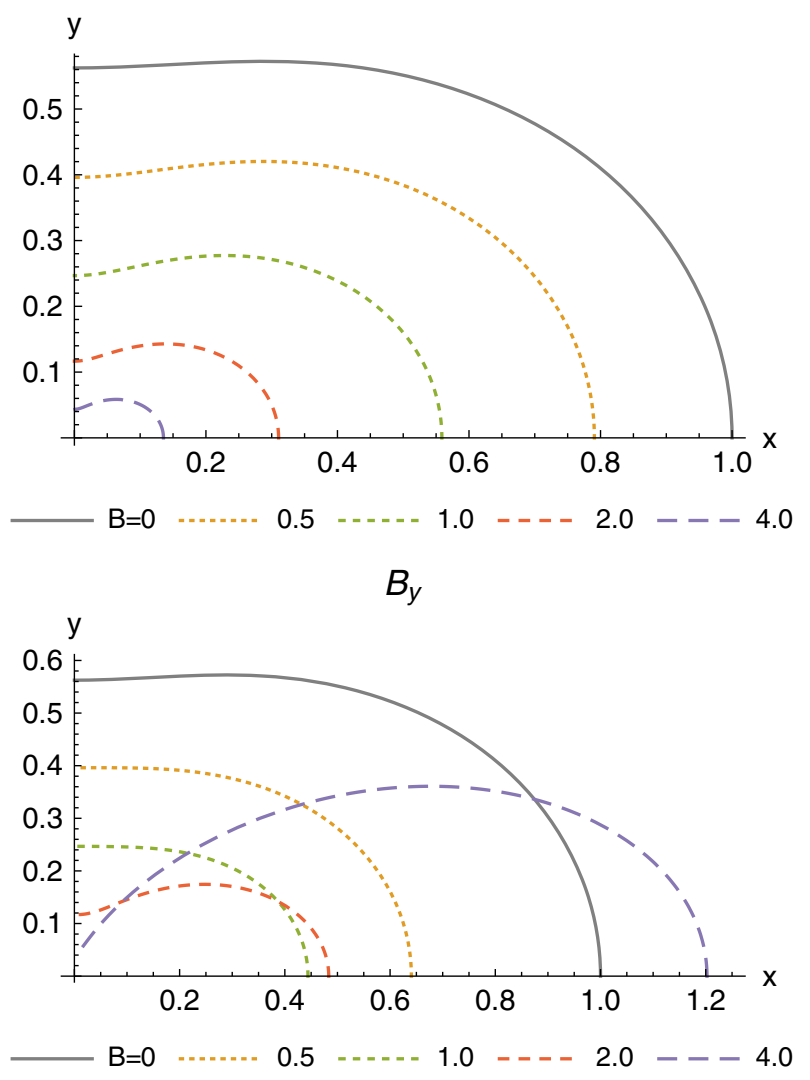

$B_{z}$

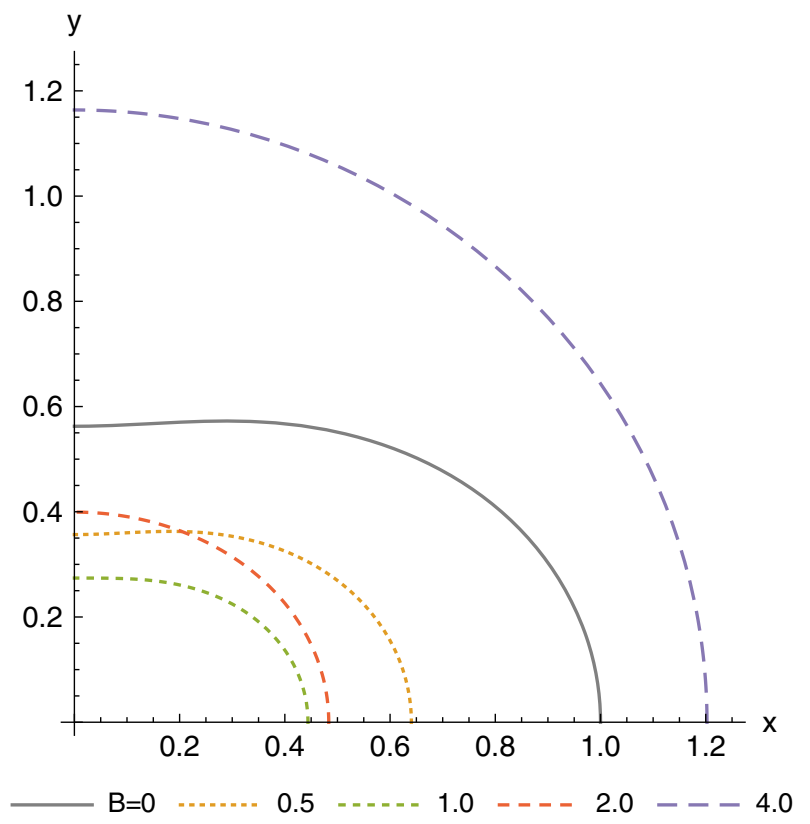

FIG. 6. Polar diagrams of the unpolarized cross section $d \sigma / d \Omega$ in the scattering plane for spinor QED with various background magnetic field strength (in units of $B_{c}$ ) and three orientations of the magnetic field (coordinates as in Fig. 1, cross section normalized to forward scattering at $B=0$ ). Top: $\mathbf{B}$ in $x$ direction, i.e., parallel to the collision axis; center: $\mathbf{B}$ in $y$ direction, in the scattering plane and orthogonal to the collision axis; bottom: $\mathbf{B}$ in $z$ direction, orthogonal to the scattering plane. (Only one quadrant of the polar plot is shown.) 
[1] H. Euler and B. Kockel, Über die Streuung von Licht an Licht nach der Diracschen Theorie, Naturwissenschaften 23, 246 (1935).

[2] H. Euler, Über die Streuung von Licht an Licht nach der Diracschen Theorie, Ann. Phys. (Berlin) 418, 398 (1936).

[3] A. Akhieser, L. Landau, and I. Pomeranchook, Light by light scattering, Nature (London) 138, 206 (1936).

[4] V. B. Berestetskii, L. P. Pitaevskii, and E. M. Lifshitz, Quantum Electrodynamics, Landau and Lifshitz: Course in Theoretical Physics, 2nd ed. (Butterworth-Heinemann, Oxford, 1982), Vol. 4.

[5] W. Heisenberg and H. Euler, Consequences of Dirac's theory of positrons, Z. Phys. 98, 714 (1936).

[6] G. V. Dunne, Heisenberg-Euler effective Lagrangians: Basics and extensions, in From Fields to Strings: Circumnavigating Theoretical Physics: Ian Kogan Memorial Collection, edited by M. Shifman, A. Vainshtein, and J. Wheater (World Scientific, Singapore, 2004) pp. 445-522.

[7] G. V. Dunne, The Heisenberg-Euler effective action: 75 years on, Int. J. Mod. Phys. A 27, 1260004 (2012).

[8] W. Dittrich, The Heisenberg-Euler Lagrangian as an example of an effective field theory, Int. J. Mod. Phys. A 29, 1430052 (2014).

[9] K. Scharnhorst, Photon-photon scattering and related phenomena. Experimental and theoretical approaches: The early period, arXiv:1711.05194.

[10] C. Itzykson and J. B. Zuber, Quantum Field Theory, International Series in Pure and Applied Physics (McGrawHill, New York, 1980).

[11] Z. Bern, A. De Freitas, L. J. Dixon, A. Ghinculov, and H. L. Wong, QCD and QED corrections to light by light scattering, J. High Energy Phys. 11 (2001) 031.

[12] Y. Liang and A. Czarnecki, Photon-photon scattering: A tutorial, Can. J. Phys. 90, 11 (2012).

[13] D. d'Enterria and G. G. da Silveira, Observing Light-byLight Scattering at the Large Hadron Collider, Phys. Rev. Lett. 111, 080405 (2013); Erratum: Observing Light-byLight Scattering at the Large Hadron Collider, Phys. Rev. Lett. 116, 129901(E) (2016).

[14] M. Kłusek-Gawenda, P. Lebiedowicz, and A. Szczurek, Light-by-light scattering in ultraperipheral $\mathrm{Pb}-\mathrm{Pb}$ collisions at energies available at the CERN Large Hadron Collider, Phys. Rev. C 93, 044907 (2016).

[15] A. Rebhan and G. Turk, Polarization effects in light-by-light scattering: Euler-Heisenberg versus Born-Infeld, Int. J. Mod. Phys. A 32, 1750053 (2017).

[16] J. Ellis, N.E. Mavromatos, and T. You, Light-by-Light Scattering Constraint on Born-Infeld Theory, Phys. Rev. Lett. 118, 261802 (2017).

[17] H. Gies, F. Karbstein, C. Kohlfürst, and N. Seegert, Photonphoton scattering at the high-intensity frontier, Phys. Rev. D 97, 076002 (2018).

[18] M. Aaboud et al. (ATLAS Collaboration), Evidence for light-by-light scattering in heavy-ion collisions with the ATLAS detector at the LHC, Nat. Phys. 13, 852 (2017).

[19] D. d'Enterria (CMS Collaboration), Evidence for lightby-light scattering in ultraperipheral $\mathrm{PbPb}$ collisions at $\sqrt{s_{N N}}=5.02 \mathrm{TeV}$, arXiv:1808.03524.

[20] D. E. Kharzeev, L. D. McLerran, and H. J. Warringa, The effects of topological charge change in heavy ion collisions:
"Event by event $P$ and $C P$ violation", Nucl. Phys. A803, 227 (2008).

[21] A. Bzdak and V. Skokov, Event-by-event fluctuations of magnetic and electric fields in heavy ion collisions, Phys. Lett. B 710, 171 (2012).

[22] W.-T. Deng and X.-G. Huang, Event-by-event generation of electromagnetic fields in heavy-ion collisions, Phys. Rev. C 85, 044907 (2012).

[23] K. Itakura, Strong-field physics as a new probe of early-time dynamics in heavy-ion collisions, J. Phys. Conf. Ser. 422, 012029 (2013).

[24] S. L. Adler, Photon splitting and photon dispersion in a strong magnetic field, Ann. Phys. (N.Y.) 67, 599 (1971).

[25] R. Karplus and M. Neuman, The scattering of light by light, Phys. Rev. 83, 776 (1951).

[26] V. Weisskopf, Über die Elektrodynamik des Vakuums auf Grund der Quantentheorie des Elektrons, Kong. Dan. Vid. Selsk. Mat.-fys. Medd. XIV/6, 1 (1936).

[27] V. S. Vanyashin and M. V. Terentev, The vacuum polarization of a charged vector field, Zh. Eksp. Teor. Fiz. 48, 565 (1965) [Sov. Phys. JETP 21, 375 (1965)].

[28] J. Ambjorn and P. Olesen, Antiscreening of large magnetic fields by vector bosons, Phys. Lett. B 214, 565 (1988).

[29] M. N. Chernodub, Superconductivity of QCD vacuum in strong magnetic field, Phys. Rev. D 82, 085011 (2010).

[30] Y. Hidaka and A. Yamamoto, Charged vector mesons in a strong magnetic field, Phys. Rev. D 87, 094502 (2013).

[31] M. N. Chernodub, Comment on charged vector mesons in a strong magnetic field, Phys. Rev. D 89, 018501 (2014).

[32] J. S. Schwinger, On gauge invariance and vacuum polarization, Phys. Rev. 82, 664 (1951).

[33] W. Dittrich and H. Gies, Probing the quantum vacuum. Perturbative effective action approach in quantum electrodynamics and its application, Springer Tracts Mod. Phys. 166, 1 (2000).

[34] C. Schubert, Perturbative quantum field theory in the string inspired formalism, Phys. Rep. 355, 73 (2001).

[35] A. Samsonov, Magnetic moment of the $\rho$ meson in QCD sum rules: $\alpha_{s}$ corrections, J. High Energy Phys. 12 (2003) 061.

[36] D. Djukanovic, M. R. Schindler, J. Gegelia, and S. Scherer, Quantum Electrodynamics for Vector Mesons, Phys. Rev. Lett. 95, 012001 (2005).

[37] F. Jegerlehner and A. Nyffeler, The muon g-2, Phys. Rep. 477, 1 (2009).

[38] V. V. Skalozub, The vacuum polarization of the charged vector field in the renormalized theory, Yad. Fiz. 21, 1337 (1975).

[39] F. Přeuřil and J. Hořejší, Effective Euler-Heisenberg Lagrangians in models of QED, J. Phys. G 45, 085005 (2018).

[40] P. V. Buividovich, M. N. Chernodub, D. E. Kharzeev, T. Kalaydzhyan, E. V. Luschevskaya, and M. I. Polikarpov, Magnetic-Field-Induced Insulator-Conductor Transition in SU(2) Quenched Lattice Gauge Theory, Phys. Rev. Lett. 105, 132001 (2010).

[41] V. V. Braguta, P. V. Buividovich, M. N. Chernodub, A. Yu. Kotov, and M. I. Polikarpov, Electromagnetic superconductivity of vacuum induced by strong magnetic field: Numerical evidence in lattice gauge theory, Phys. Lett. B 718, 667 (2012). 
[42] E. V. Luschevskaya, O. E. Solovjeva, and O. V. Teryaev, Determination of the properties of vector mesons in external magnetic field by quenched $S U(3)$ lattice QCD, J. High Energy Phys. 09 (2017) 142.

[43] G. S. Bali, B. B. Brandt, G. Endrődi, and B. Gläßle, Meson masses in electromagnetic fields with Wilson fermions, Phys. Rev. D 97, 034505 (2018).
[44] Y. Liao, Multi-photon decays of the Higgs Boson in standard model: Leading terms from Heisenberg-Euler effective Lagrangian, Phys. Rev. D 86, 117302 (2012).

[45] J. S. Heyl and L. Hernquist, Analytic form for the effective Lagrangian of QED and its application to pair production and photon splitting, Phys. Rev. D 55, 2449 (1997). 